\title{
LBT Enhancement for LTE-LAA Fair Coexistence
}

\author{
Amsatou Mbengue $^{1}$ and Yongyu Chang ${ }^{2}$ \\ WT\&T Lab, Beijing University of Posts and Telecommunications, P. R. China \\ ${ }^{1}$ Email:amsa@bupt.edu.cn, ${ }^{2}$ Email:yychang@bupt.edu.cn
}

\begin{abstract}
A common approach to increase wireless network capacity is to use additional spectrum. LTE in the unlicensed spectrum band has been introduced in the release 13 of 3GPP as Licensed Assisted Access (LAA). The LTE technology in the unlicensed band is enabled by Listen Before Talk (LBT) procedure for fair coexistence with other wireless technologies (i.e., Wi-Fi) operating in the same spectrum. LBT based on binary exponential back-off known as LBT category 4 can achieve a fair coexistence in low buffer but presents some limitations under full buffer evaluation. In this paper we propose different LBT mechanisms and evaluate their performances based on 3GPP coexistence scenarios (LAA/Wi-Fi, LAA/LAA). The simulation results show that the LBT category 4 with Optimal Constant Contention Window $(O C C W)$ and the proposed fixed periodic LBT $(P-L B T)$ can achieve better performance under saturation state even better than Wi-Fi only coexistence.
\end{abstract}

Keywords: LAA, LBT, LTE, Wi-Fi, LBT- OCCW, P-LBT

\section{Introduction}

To satisfy the high demand of traffic service, operators provide a wide range of network possibilities. They focus on two options: one is to offload their traffic to the WiFi networks another alternative is to use the LTE in the unlicensed band. The unlicensed band is a shared spectrum for different technologies a fairness coexistence with other technologies and with other operators in the same spectrum is the key challenge for industries and regulation organizations [1]. Many works have been done for possible coexistence between wireless technologies in the unlicensed spectrum. [2-3] show that if a Wi-Fi technology coexists with LTE technology in the unlicensed bands, the Wi-Fi performance would be severely degraded. An overview of the LTE in the unlicensed band is presented in [4],[5] where the authors described the trends of this technology with possible deployment and modification requirement in LTE side to well coexist with WiFi.

The performance of TD-LTE with Wi-Fi presented in [6] shows that Wi-Fi performance is less affected during the LTE uplink transmission than the downlink operation. [7-8] present a coexistence gap approach to enable LTE in the TV white space and possible deployment with ZigBee under 2.4Ghz. In [9] detect and avoid mechanisms are proposed for mitigating interference to enable ultra-wideband (UWB) and WiMAX to operate in the same frequency band. With [10] the author focused on the channel selection functionality for LTE in unlicensed band (LTE-U) to enable cells to decide the most appropriate channel to use for downlink traffic in the unlicensed band. Both LTE and WiFi use OFDM with different symbol length and subcarrier band, in [11] the author proposes a protocol stack, a fusion between LTE and 802.11 to enable LTE decoding WiFi signal for better coexistence. In [12] the authors analyzed the effectiveness of LBT and interference-award channel selection between LAA and Wi-Fi with different operators. The simulation results showed that a random channel selection can be adopted in case of low network density deployment. LTE can operate in different bandwidth, [13] presented 
a possible way to deploy LAA with different channel bandwidth. The experimental results showed that using small bandwidth $(1.4 / 3 / 5 / 10 \mathrm{MHz})$ may have large impact in the Wi-Fi performance. In [14] the authors proposed an investigation of adaptive p-persistent carrier sense multiple access with collision avoidance (CSMA) scheme for LTE-U based dynamic adjustment probability according to the interference measurement. The numerical results show that this method can achieve better performance compared to the static CSMA approach.

Carrier aggregation between licensed and unlicensed band can offer significant improvements in terms of capacity and Quality of Service (QoS), [15] shows that with $2 \mathrm{Tx}-2 \mathrm{Rx}$ over $20 \mathrm{MHz}$, LAA can provide a downlink throughput up to 60Mbps, [16] develops a possible multicarrier LAA to increase this throughput with less impact. From synchronous to asynchronous model, different LBT mechanisms were proposed in [17], it showed that asynchronous LBT can achieve good performance with less complexity implementation. In [18] the authors developed an analytic method to evaluate the coexistence of LTE and Wi-Fi in the unlicensed band of 5GHz. This analysis showed that LAA nodes can transmit with two different data rates (high and low). Due to the Channel Quality Indicator (CQI) report by the UEs to eNBs, collision occur only during the high data rate transmission. With constant window size, eNBs only adapt the Modulation Coding Scheme (MCS) according to the channel quality report.

In combination with a judicious energy detection threshold and contention windows, LAA based on LBT offer better throughput and less degradation of the Wi-Fi performance [19]. In this point of view, [20] presents the effect of the transmit power of the station and the Clear Channel Assessment (CCA) energy detection threshold, the evaluation results show that increasing the transmit power with appropriate CCA level can improve the network throughput. [21] presents an optimal radio access procedure for LAA to switch between schedule and random schemes.

Most of the works in LAA proposed by 3GPP (LBT category 4) present a faire coexistence with Wi-Fi in low buffer evaluation. How LAA can affect Wi-Fi network or another LAA network under saturation evaluation? In this work we present two LBT mechanisms, a modified LBT category 4 with an optimal constant contention window and a periodic waiting time LBT (P-LBT) to evaluate the performances of LAA/Wi-Fi and LAA/LAA coexistence under full buffer in indoor scenario. It is observed that LBT category 4 is not suitable for LAA under full buffer situation, LAA nodes keep transmitting while Wi-Fi nodes waiting for the channel to be free. Based on the 3GPP indoor scenario, the proposed LBT mechanisms allow both networks to operate with more fair behaviors even better than two Wi-Fi or LAA operators' coexistence.

The rest of this paper is organized as follows. In Section II we present the proposed LBT principles and enhancement. Section III presents the simulation scenarios and performance evaluation. We conclude this work in section IV.

\section{LBT Enhancement}

Different coexistence mechanisms were proposed in the beginning of LTE in the unlicensed band. In the one hand a time sharing approach between LTE and Wi-Fi, this method called LTE-U (non 3GPP standard) is based on duty-cycle coexistence. The LTE$\mathrm{U}$ system is tuned on during a period of time and turned off for the rest of the cycle [22], [23]. In the other hand, two standardized access procedures were proposed by 3GPP: LTE Wi-Fi link Aggregation (LWA) and LAA. The LWA consists of transmitting the LTE signal over a Wi-Fi channel while the LAA consists of a coexistence between different technologies based on a carrier sense access mechanism. The LAA gains more attraction compared to the LWA for industries and laboratories. LBT principle is already used by the IEEE 802.11 networks, it is very similar to the LBT-Category 4 proposed by $3 \mathrm{GPP}$. 


\subsection{Energy Detection Threshold}

In the IEEE 802.11 network the listen before talk is known as Distribution Coordination Function (DCF). DCF is a random access scheme based on CSMA. Before transmitting any data Wi-Fi nodes need to know whether the channel is free or not; two techniques are used for this process. One is the energy detection CCA where the nodes sense the energy level of the channel to determine whether it is busy or free. For Wi-Fi technology the energy level is fixed to $-62 \mathrm{dBm}$ at $20 \mathrm{MHz}$. The second technique is the preamble CCA, where the network packets are transmitted with a short training field (STF) and a long training field (LTF), a high correlation value means the presence of WiFi signal, the energy level in this approach is fixed to $-82 \mathrm{dBm}$ over $20 \mathrm{MHz}$ channel bandwidth. LTE in the unlicensed band needs a mechanism that can take in account this principle to avoid interfering to the Wi-Fi network or another LAA network. LTE is not able to decode a preamble Wi-Fi signal; the energy detection approach can be used to reduce the complexity of the implementation. We adopt an energy detection approach at $82 \mathrm{dBm}$, in this way the proposed LBT can detect whether the channel is free or not.

\subsection{LBT Category 4 with Optimal Constant Contention Window}

As specified in 3GPP technical report [24], the category 4 of LBT is very similar to the 802.11 DCF. The LBT category 4 mechanism needs to sense the channel free for at least $34 \mu \mathrm{s}$ as an initial Clear Channel Assessment (iCCA) followed by the extended clear channel assessment (eCCA). No QoS mechanism was defined in the LBT category 4. Similar to 802.11 DCF, LBT category 4 use a binary exponential back-off function in case of fail transmission (delay ACK/NACK) and reset its contention window size in case of successful transmission. This approach may be not optimal in saturation network; LAA users will keep transmitting while Wi-Fi nodes keep waiting for the channel to be free. This situation is similar to the LTE/Wi-Fi coexistence without LBT mechanism under low buffer evaluation. We proposed a modified version of the LBT category 4 with an Optimal Constant Contention Window (OCCW) to get the maximum user throughput and less channel access delay. This principle was investigated in [25], [26] for Wi-Fi only network.

Even specified in the 3GPP LAA TR, the contention windows $(\mathrm{CW})$ size of LBT category 4 should be variable, however most of the analytical works in LAA/Wi-Fi consider a fixed contention windows (LBT category 3). The reason that LAA adopts a fixed $\mathrm{CW}$ in some cases is that collision in LAA network does not mean failed transmission. The variation of the contention window size occurs only in high interference. In case of high network traffic (full buffer evaluation), the IEEE DCF Bianchi' model (Wi-Fi/Wi-Fi coexistence model) can be used to optimize the network performances of LBT category 4 . Let denote $b i, k$ the stationary probability of the Markov model [25]:

$$
b_{i, k}=\frac{W_{i}-k}{W_{i}} b_{i, 0} \quad i=[0, m], k \in\left[0, W_{i}-1\right]
$$

where $W i$ denotes the contention window size and $\mathrm{k}$ the random back-off value.

$$
b_{0,0}=\frac{2(1-2 p)(1-p)}{(1-2 p)(W+1)+p W\left(1-(2 p)^{m}\right)}
$$

The probability that a station transmits in a random slot is given by:

$$
\tau=\frac{2}{W+1}
$$


The probability for at least one transmission in a considered slot time is given by:

$$
P_{t r}=1-(1-\tau)^{n}
$$

where $n$ represent the number of nodes. The probability for a successful transmission for at least one nodes is represented by $P s$

$$
P_{s}=\frac{n \tau(1-\tau)^{n-1}}{P_{t r}}=\frac{n \tau(1-\tau)^{n-1}}{1-(1-\tau)^{n}}
$$

The throughput is given by equation (6) where $P$ represents the payload information transmitted in a slot time. $T s$ and $T c$ represent respectively the time for successful transmission and collision time and $\sigma$ denotes the CCA slot time.

$$
S=\frac{E[P]}{T_{s}-T_{c}+\frac{\sigma\left(1-P_{t r}\right) / P_{t r}+T_{c}}{P_{s}}}
$$

The optimal contention window depends on the number of nodes trying to access to the channel, since the transmission duration, the slot time and the propagation delay are supposed to be constant. We maximize the throughput $S$ by derivative respect to $\tau$ and impose to zero:

$$
(1-\tau)^{n} \approx 1-n \tau+\frac{(1-\tau)^{n}}{2} \tau^{2}
$$

Then an approximate of $\tau$ is given by:

$$
\tau \approx \frac{1}{n \sqrt{\frac{2 T_{c}}{\sigma}}}
$$

Replacing $\tau$ in equation (3), we obtain the optimal contention window Woptimal

$$
W_{\text {optimal }} \approx n \sqrt{\frac{2 T_{c}}{\sigma}}
$$

This analytical model, under saturation situation, provides an optimal contention window. With LBT category 4, the algorithm generates a random back-off value between zero and the optimal window. Having the optimal window can give an approximation of timing period in case of static LBT design. In the next paragraph we propose a periodic LBT to optimize the transmission delay.

\subsection{Periodic LBT (P-LBT) Mechanism}

LTE adapts its modulation coding based on CQI feedback this function makes LTE very strong against noisy channel. The deployment of LTE in the unlicensed band based on LBT category 4 can present some drawbacks in case of saturate network. A contention window mechanism (LBT-category 4 and 802.11 DCF), reset its contention window size in case of successful transmission. When we adapt this approach for LTE in the unlicensed band, LAA network can resist better than the Wi-Fi in case of high interference level, thus LAA eNB can reset their contention window and have more opportunity to access to the channel than Wi-Fi AP.

In the rest of this section we present the second proposition mechanism for fair coexistence between LAA/Wi-Fi and LAA/LAA. Figure 1 shows a comparison between 
the different frame structures. The proposed method is similarity to LBT category 4, a fixed window size is adopted even after successful transmission. LAA eNB can access to the channel during a constant transmission opportunity time $(10 \mathrm{~ms})$ this time is equivalent to the LTE frame period, then LAA nodes need to wait for a fixe time of 5ms (sub-frame timing) even if the channel is free. A defer time of $10 \mu$ s is adopted after data transmission to release the channel. We chose a larger CCA slot time of $50 \mu$ s similar to $802.11 \mathrm{~b}$. In this approach LAA nodes adapt more fair behavior in the unlicensed spectrum band than LBT-category 4.

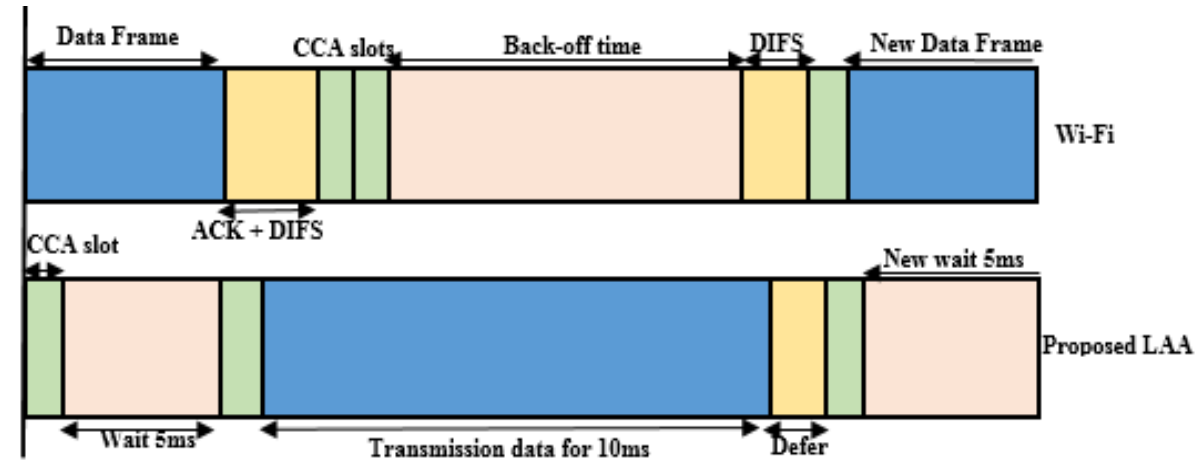

Figure 1. Frame Structure of Wi-Fi and Proposed P-LBT

\section{Performance Results}

This section presents detailed performances of the different coexistence mechanisms described in section II. The evaluation is based on the 3GPP simulation scenario defined in [24] with full buffer evaluations using NS3 simulation platform. The first step of the simulation procedure is done with two Wi-Fi operators in the same $20 \mathrm{MHz}$ spectrum. The recorded values from this scenario are used as a reference to evaluate the impact of the LAA network to the Wi-Fi under low buffer situation. The second step of the simulation records the performances of LAA/Wi-Fi and LAA/LAA coexistence based on LBT category 4 and compared them to the proposed LBT methods. We adopt an energy detection threshold of $-82 \mathrm{dBm}$ to allow LAA nodes to consider Wi-Fi signal with lower energy level. The main aspects we evaluate are the user throughput and the latency. Figure 2 presents the simulation scenario proposed in 3GPP technical report [24].

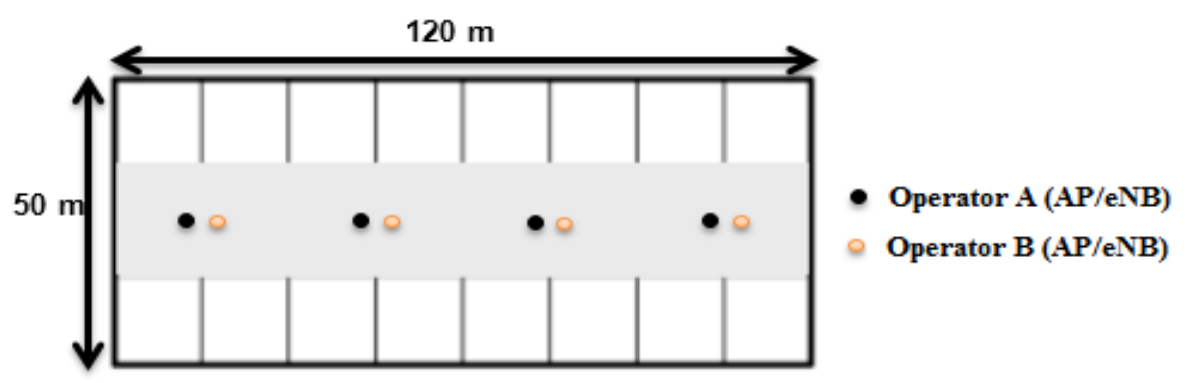

Figure 2. Indoor Scenario for LAA/ Wi-Fi and LAA/LAA Coexistence [24] 
Table 1. Simulation Parameters

\begin{tabular}{|l|l|}
\hline Parameters & Values \\
\hline LAA Antenna configuration & $2 \mathrm{Tx} 2 \mathrm{Rx}$ \\
\hline LAA / Wi-Fi CCA-ED & $-82 \mathrm{dBm}$ \\
\hline Traffic model & FTP [15] lambda 2.5, UDP Full buffer \\
\hline Slot time & Cat4 10us, P-LBT 50us \\
\hline Defer time & Cat4 34us, P-LBT 10us \\
\hline bandwidth per carrier & $20 \mathrm{MHz}$ \\
\hline Number of UE & 40,20 per operator, 5 per AP/eNB \\
\hline
\end{tabular}

\subsection{LAA-Wi-Fi LAA-LAA Coexistence with LBT Category 4}

Different simulation works have been done in low buffer scenario using 3GPP traffic model and their results show a fair coexistence based on LBT category 4. In Figure 3 we present this possibility under low-buffer condition, the Wi-Fi performances are almost the same when it coexists with LAA network or another Wi-Fi operator. This result coincides with [27]. In the next phase of this paper we evaluate the performances of LAA/Wi-Fi and LAA/LAA coexistence scenarios under full-buffer situation. When two Wi-Fi operators coexist in this scenario, Figure 4 shows that under full buffer situation, both operators impact each other and can achieve a throughput between 1.7 to $4 \mathrm{Mbps}$.

In LAA/Wi-Fi coexistence based on the simulation parameters in Table 1 using LBT category 4 the performance of the Wi-Fi network is severely impacted; more than $75 \%$ of the Wi-Fi users have a throughput less than 0.5Mbps. In this case LAA network keep using the channel while Wi-Fi users keep waiting to the channel to be free. The main reason is that LAA users are able to report the channel quality by sending a CQI message to the eNB thus the eNB can adapt its modulation code according to the interference level. After a successful transmission, LAA based on LBT category 4 reset its CW to the minimum value (15), lower $\mathrm{CW}$ provides LAA eNBs less waiting time before the next channel access tentative.

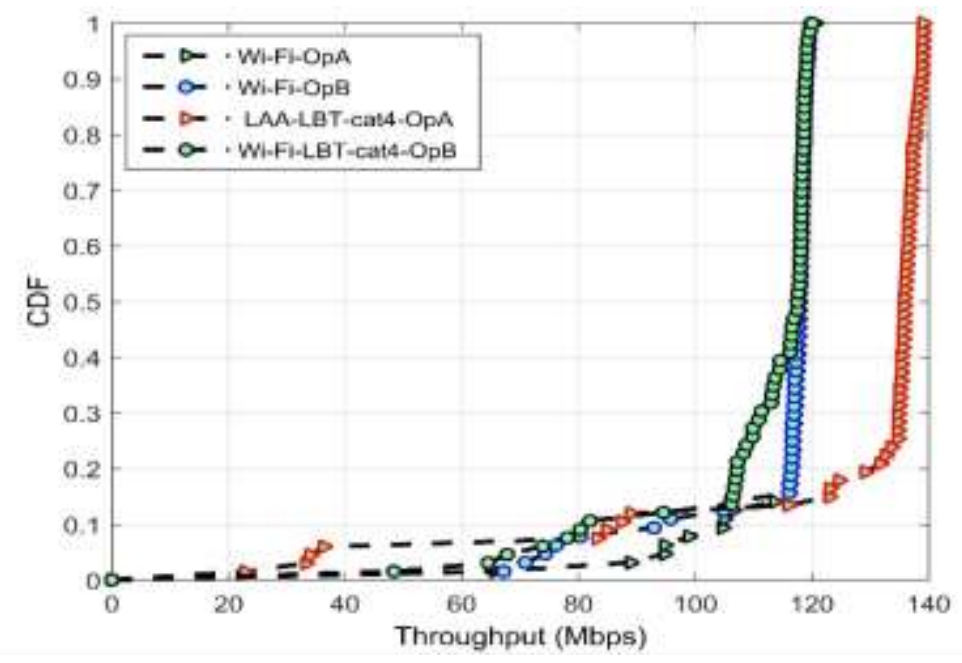

Figure 3. LAA-Wi-Fi Throughput with LBT Category 4 at Low Buffer 


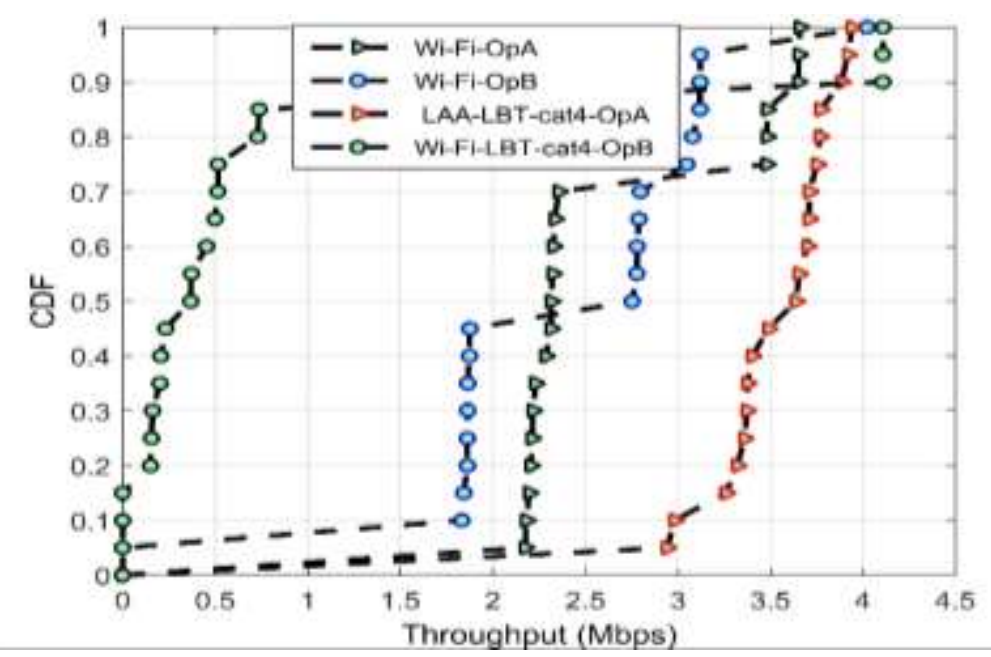

Figure 4. Wi-Fi/Wi-Fi and LAA/Wi-Fi Throughput with LBT Category 4

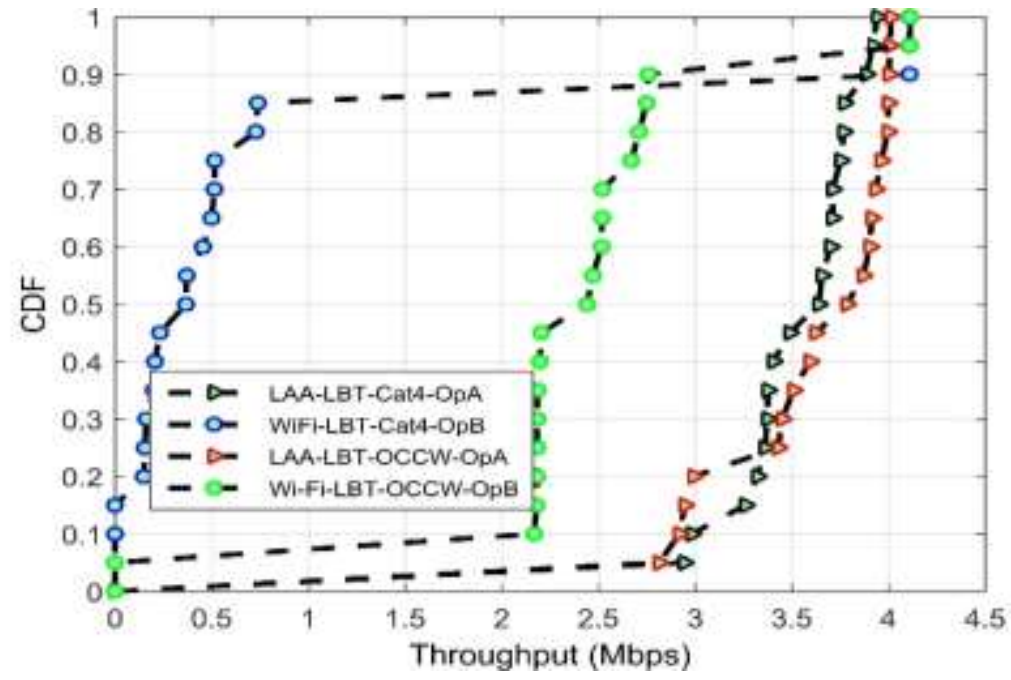

Figure 5. LAA-Wi-Fi Throughput with LBT-OCCW and LBT Category 4

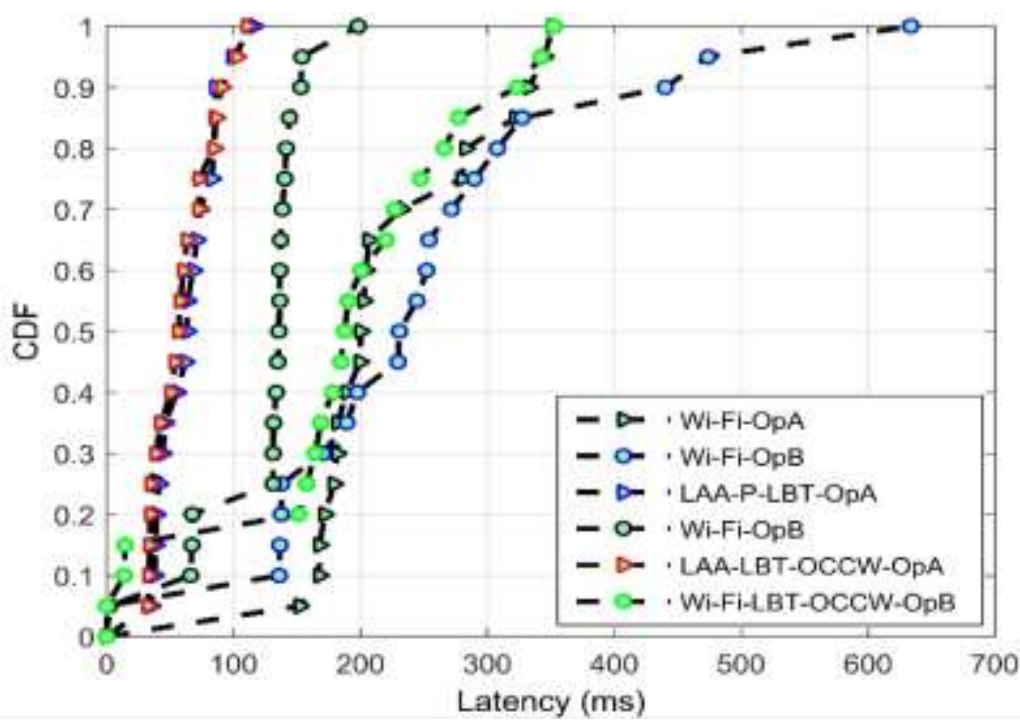

Figure 6. Wi-Fi-Wi-Fi and Wi-Fi-LAA Latency with Different LBT 


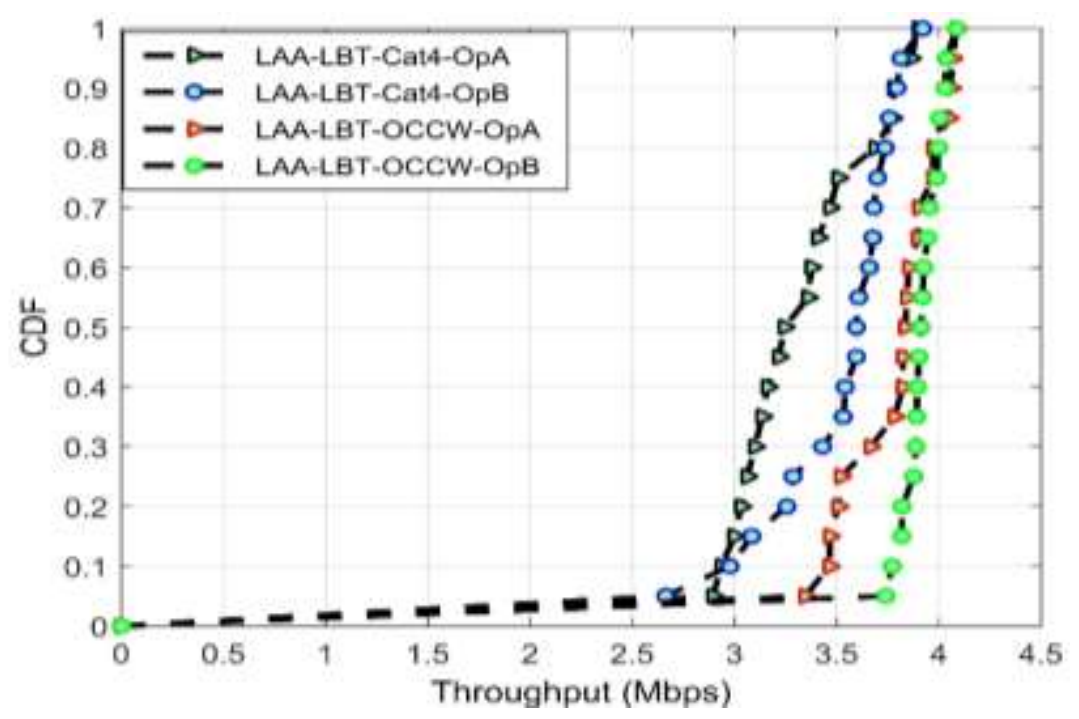

Figure 7. LAA-LAA Throughput with LBT Category 4 and LBT-OCCW

\subsection{LAA-Wi-Fi LAA-LAA Coexistence with LBT-OCCW}

The simulation results in Figure 5 show that with an optimal constant contention window, LAA/Wi-Fi coexistence can achieve better perform compared to LAA based on general LBT category 4 . Using the simulation parameters as Wi-Fi-like all Wi-Fi users can achieve a throughput higher than $2 \mathrm{Mbps}$. The performance of the LAA network is also improved with better throughput and less latency, in Figure 6 we present the latency of the different coexistence methods between LAA and Wi-Fi. The LBT design based on OCCW can perform better than two Wi-Fi operators' coexistence. Figure 7 presents the performance comparison between two operators using LBT category 4 and LBT-OCCW. In this case the latency of both operators is also improved. In the next part of this work we present the simulation results of our second proposition, P-LBT mechanism.

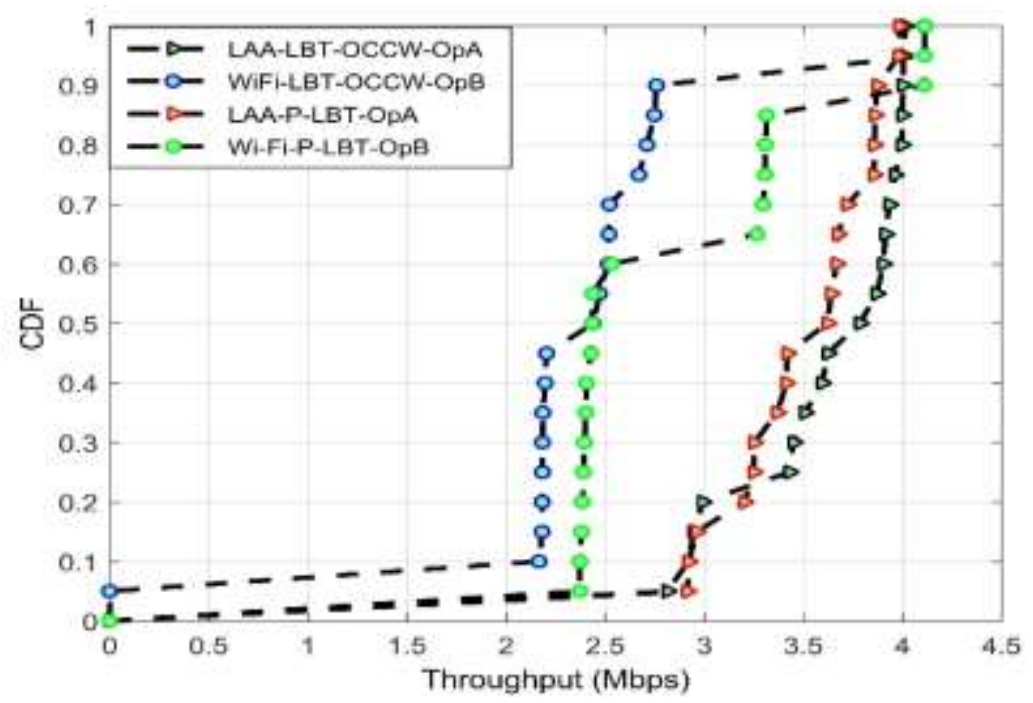

Figure 8. LAA-Wi-Fi Throughput with LBT-OCCW and P-LBT

\subsection{LAA-Wi-Fi, LAA-LAA Coexistence with P-LBT}

As we describe in Figure 1, the proposed P-LBT mechanism is evaluated in the same scenario as the preview methods. The simulation results presented in Figure 8 show that 
the P-LBT compare to the LBT-OCCW performs with more fairness. The user throughput in slightly less than the LAA network but the Wi-Fi users achieve higher throughput even better than the case of two Wi-Fi operators' coexistence scenario in Figure 3.

In Figure 6, the latency of the Wi-Fi users is significantly improved compare to the case of LAA/Wi-Fi coexistence based on LBT-OCCW or LBT category 4 or in the case of two Wi-Fi operators. In LAA/LAA coexistence scenario with P-LBT, the user throughput is less than in the first proposition see Figure 9.

Figure 10 shows that using the proposed P-LBT, 100\% of LAA users have a latency less than $100 \mathrm{~ms}$ while $60 \%$ have a latency less than $100 \mathrm{~ms}$ in case of LBT category 4 . PLBT has also less latency compared to LBT-OCCW.

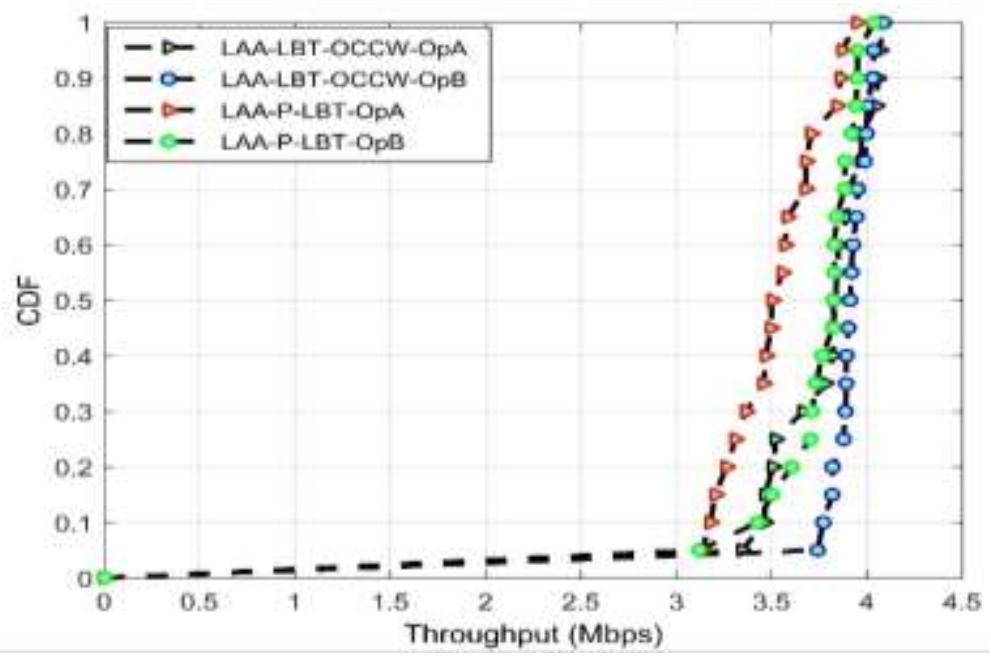

Figure 9. LAA-LAA Throughput with P-LBT and LBT-OCCW

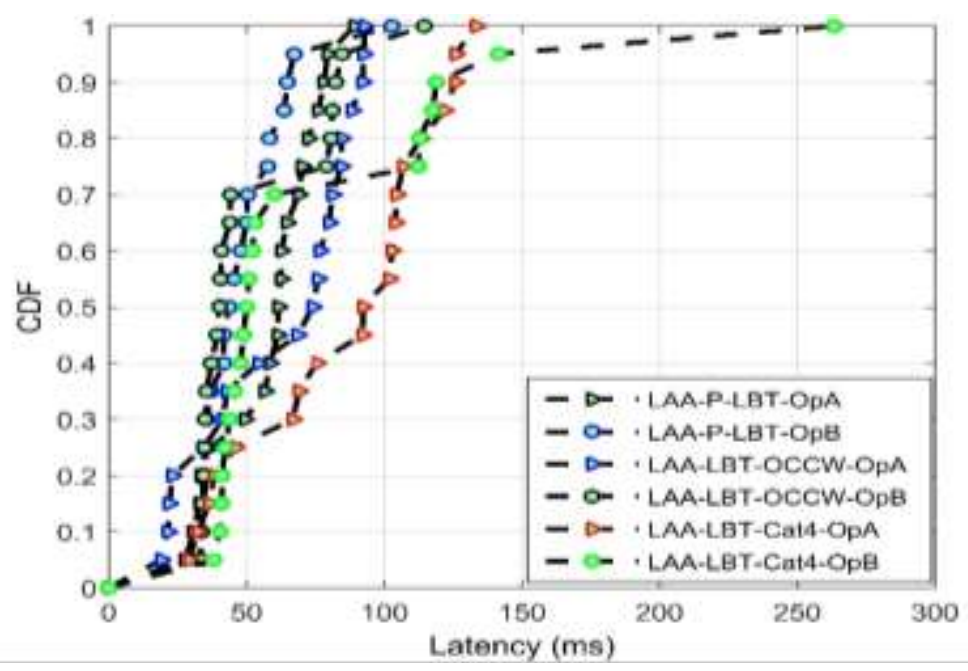

Figure 10. Wi-Fi-Wi-Fi and Wi-Fi-LAA Latency with different LBT

\section{Conclusion}

In this paper we present different coexistence mechanisms for LTE operating in the unlicensed spectrum band. For more fair coexistence we proposed an enhancement with an optimal constant contention window in the saturation state and a second LBT procedure called P-LBT with fixed channel sensing period. The simulation results show that under full buffer situation, the proposed LBT based on constant optimal contention window operates better than the LBT category 4 proposed in 3GPP standard. The last 
proposition (P-LBT) presents more fairness, both LAA and Wi-Fi can share the same unlicensed spectrum with less impact to each other.

\section{References}

[1] A. Pyattaev, K. Johnsson, S. Andreev and Y. Koucheryavy, "3GPP LTE traffic offloading onto WiFi Direct", in Wireless Communications and Networking Conference Workshops (WCNCW), 2013 IEEE, (2013), pp. 135-140.

[2] F. Liu, E. Bala, E. Erkip, M. C. Beluri and R. Yang, "Small-Cell Traffic Balancing Over Licensed and Unlicensed Bands", Vehicular Technology, IEEE Transactions on, vol. 64, (2015), pp. 5850-5865.

[3] A. V. Kini, L. Canonne-Velasquez, M. Hosseinian, M. Rudolf and J. Stern-Berkowitz, "Wi-Fi-LAA coexistence: Design and evaluation of Listen Before Talk for LAA", in 2016 Annual Conference on Information Science and Systems (CISS), (2016), pp. 157-162.

[4] Z. Ran, W. Miao, L. X. Cai, Z. Zhongming and S. Xuemin, "LTE-unlicensed: the future of spectrum aggregation for cellular networks", Wireless Communications, IEEE, vol. 22, (2015), pp. 150-159.

[5] M. D. Foegelle, "Coexistence of LTE-U and LAA in a Wi-Fi world", in 2016 10th European Conference on Antennas and Propagation (EuCAP), (2016), pp. 1-5.

[6] N. Rupasinghe and I. Guvenc, "Licensed-assisted access for WiFi-LTE coexistence in the unlicensed spectrum”, in Globecom Workshops (GC Wkshps), (2014), pp. 894-899.

[7] M. Beluri, E. Bala, D. Yuying, R. D. Girolamo, M. Freda, J. L. Gauvreau, S. Laughlin, D. Purkayastha and A. Touag, "Mechanisms for LTE coexistence in TV white space", in Dynamic Spectrum Access Networks (DYSPAN), 2012 IEEE International Symposium on, (2012), pp. 317-326.

[8] I. Parvez, N. Islam, N. Rupasinghe, A. I. Sarwat and Ven, "LAA-based LTE and ZigBee coexistence for unlicensed-band smart grid communications", in SoutheastCon 2016, (2016), pp. 1-6.

[9] S. M. Mishra, R. W. Brodersen, S. T. Brink and R. Mahadevappa, "Detect and avoid: an ultrawideband/WiMAX coexistence mechanism [Topics in Radio Communications]", Communications Magazine, IEEE, vol. 45, (2007), pp. 68-75.

[10] J. Hoiyoon, U. Jungsun, Y. Sungjin and P. Seungkeun, "Throughput enhancement with carrier selection for LTE in unlicensed band", in Information and Communication Technology Convergence (ICTC), 2015 International Conference on, (2015), pp. 837-839.

[11] S. Hao and F. Xuming, "A spectrum etiquette protocol and interference coordination for LTE in unlicensed bands (LTE-U)", in Communication Workshop (ICCW), 2015 IEEE International Conference on, (2015), pp. 2338-2343.

[12] A. M. Voicu, L. Simic and M. Petrova, "Coexistence of pico- and femto-cellular LTE-unlicensed with legacy indoor Wi-Fi deployments", in Communication Workshop (ICCW), 2015 IEEE International Conference on, (2015), pp. 2294-2300.

[13] J. Yubing, S. Chao-Fang, B. Krishnaswamy and R. Sivakumar, "Coexistence of Wi-Fi and LAA-LTE: Experimental evaluation, analysis and insights", in Communication Workshop (ICCW), 2015 IEEE International Conference on, (2015), pp. 2325-2331.

[14] J. Baoan, "An adaptive p-persistent CSMA scheme for LTE in unlicensed bands", in Wireless Communications \& Signal Processing (WCSP), 2015 International Conference on, (2015), pp. 1-5.

[15] R. Ratasuk, N. Mangalvedhe and A. Ghosh, "LTE in unlicensed spectrum using licensed-assisted access", in Globecom Workshops (GC Wkshps), 2014, (2014), pp. 746-751.

[16] J. Liu and G. Shen, "Performance of Multi-Carrier LBT Mechanism for LTE-LAA", in 2016 IEEE 83rd Vehicular Technology Conference (VTC Spring), (2016), pp. 1-5.

[17] A. Bhorkar, C. Ibars, A. Papathanassiou and P. Zong, "Medium access design for LTE in unlicensed band", in Wireless Communications and Networking Conference Workshops (WCNCW), 2015 IEEE, (2015), pp. 369-373.

[18] C. Cheng, R. Ratasuk and A. Ghosh, "Downlink Performance Analysis of LTE and WiFi Coexistence in Unlicensed Bands with a Simple Listen-Before-Talk Scheme", in Vehicular Technology Conference (VTC Spring), 2015 IEEE 81st, (2015), pp. 1-5.

[19] A. M. Baswade and B. R. Tamma, "Channel sensing based dynamic adjustment of contention window in LAA-LTE networks", in 2016 8th International Conference on Communication Systems and Networks (COMSNETS), (2016), pp. 1-2.

[20] X. Pengfei, T. Zi and J. Wu, "How loud to talk and how hard to listen-before-talk in unlicensed LTE", in Communication Workshop (ICCW), 2015 IEEE International Conference on, (2015), pp. 2314-2319.

[21] S. Y. Lien, J. Lee and Y. C. Liang, "Random Access or Scheduling: Optimum LTE Licensed-Assisted Access to Unlicensed Spectrum", IEEE Communications Letters, vol. 20, (2016), pp. 590-593.

[22] N. Rupasinghe, $\mathrm{x}, \mathrm{x} 00 \mathrm{Fc}$, ven and $\mathrm{x} 00 \mathrm{E}$, "Reinforcement learning for licensed-assisted access of LTE in the unlicensed spectrum", in Wireless Communications and Networking Conference (WCNC), 2015 IEEE, (2015), pp. 1279-1284.

[23] V. Valls, A. Garcia-Saavedra, X. Costa and D. J. Leith, "Maximizing LTE Capacity in Unlicensed Bands (LTE-U/LAA) While Fairly Coexisting With 802.11 WLANs", IEEE Communications Letters, vol. 20, (2016), pp. 1219-1222. 
[24] 3GPP, "3rd Generation Partnership Project; Technical Specification Group Radio Access Network; Study on Licensed-Assisted Access to Unlicensed Spectrum;(Release 13)", (2015).

[25] G. Bianchi, "Performance analysis of the IEEE 802.11 distributed coordination function", IEEE Journal on Selected Areas in Communications, vol. 18, (2000), pp. 535-547.

[26] W. Ruopeng, Z. Jie and Z. Xiangyi, "Performance Analysis and Optimization of IEEE 802.11 DCF with Constant Contention Window", in Computing, Communication, Control and Management, 2008. CCCM '08. ISECS International Colloquium on, (2008), pp. 407-411.

[27] R. Kwan, R. Pazhyannur, J. Seymour, V. Chandrasekhar, S. R. Saunders, D. Bevan, H. Osman, J. Bradford, J. Robson and K. Konstantinou, "Fair co-existence of Licensed Assisted Access LTE (LAALTE) and Wi-Fi in unlicensed spectrum", in Computer Science and Electronic Engineering Conference (CEEC), 2015 7th, (2015), pp. 13-18. 
International Journal of Future Generation Communication and Networking Vol. 10, No. 1 (2017) 